\title{
Controlling spin Hall effect by using a band anticrossing and nonmagnetic impurity scattering
}

\author{
T. Mizoguchi and N. Arakawa \\ Department of Physics, The University of Tokyo, 7-3-1 Hongo, Bunkyo-ku, Tokyo 113-0033, Japan
}

(Dated: March 19, 2021)

\begin{abstract}
The spin Hall effect (SHE) is one of the promising phenomena to utilize a spin current as spintronics devices, and the theoretical understanding of its microscopic mechanism is essential to know how to control its response. Although the SHE in multiorbital systems without inversion symmetry (IS) is expected to show several characteristic properties due to the cooperative roles of orbital degrees of freedom and a lack of IS, a theoretical understanding of the cooperative roles has been lacking. To clarify the cooperative roles, we study the spin Hall conductivity (SHC) derived by the linear-response theory for a $t_{2 g}$-orbital tight-binding model of the [001] surface or interface of $\mathrm{Sr}_{2} \mathrm{RuO}_{4}$ in the presence of dilute nonmagnetic impurities. We find that the band anticrossing, arising from a combination of orbital degrees of freedom and a lack of IS, causes an increase of magnitude and a sign change of the SHC at some nonmagnetic impurity concentrations. Since a similar mechanism for controlling the magnitude and sign of the response of Hall effects works in other multiorbital systems without IS, our mechanism provides an ubiquitous method to control the magnitude and sign of the response of Hall effects in some multiorbital systems by introducing IS breaking and tuning of the nonmagnetic impurity concentration.
\end{abstract}

PACS numbers: 72.25.Ba, 73.40.-c, 74.70.Pq

The spin Hall effect (SHE) has been intensively studied due to its applicability to spintronics devices and theoretical interests. In the SHE, an external electric field causes the spin current (i.e., the flow of the spin angular momentum) which is perpendicular to this field $\stackrel{1,2}{2}$. Since controlling a charge current is easier than controlling a spin current, the SHE has a great potential for spintronics devices ${ }^{3}$. In addition, it is crucial to understand the microscopic mechanism of the SHE since it gives us a deeper understanding of how to control its response, and this motivates further research.

So far, we have partially understood the microscopic mechanisms of the SHE. These are categorized into intrinsic and extrinsic mechanisms: The former is related to the electronic structure ${ }^{4}-\underline{8}$, while the latter is related to the multiple scattering of the doped impurities, ${ }^{1,2,9-12}$. We emphasize that the intrinsic contribution arises from not only the Berry curvature term ${ }^{4-7.13}$ (part of the Fermi sea term) but also the other Fermi sea term and the Fermi surface term $\$, 14-16$ which qualitatively differ from the Berry curvature term. In several transition metals (TMs) and TM oxides, the intrinsic mechanism is more important than the extrinsic mechanism because the intrinsic mechanism often gives a large response $\frac{8,15}{2}$ and because the extrinsic mechanism is negligible for the weak scattering potential of the doped nonmagnetic impurities. Note that the realization of a such weak scattering potential is shown in a first-principles calculation in some cases 17 .

In general, we can understand the origin of the intrinsic SHE by detecting how an electron acquires the AharanovBohm-like phase $\frac{18}{18}$ due to an effective flux. For that detection, it is helpful to consider the real-space motion of an electron whose first and final positions are the same because, by considering that motion, we can discuss a phase factor of the wave function of an electron; hereafter, we call such motion a process. For example, in a multiorbital TM or TM oxide with inversion symmetry (IS) $)^{8,15,19-21}$, the spin-dependent effective flux is generated by the process of using the $z$ component of the atomic spin-orbit interaction (SOI), the interorbital hopping integral between the orbitals connected by that $z$ compo- nent, and other hopping integrals [see Fig. 11a)]. In addition, we can understand the SHE in a two-dimensional electron gas without IS ${ }^{5,22,23}$, whose electronic state is described by using the Rashba-type antisymmetric $\mathrm{SOI}^{24}$ by considering the process using the Rashba-type antisymmetric SOI and the intraorbital hopping integrals [see Fig. 1(b)].

Although there are many studies about the SHE in multiorbital systems with IS (e.g., Refs. 8 and 15) or single-orbital systems without IS (e.g., Refs. 5 and 25), the characteristic properties of the SHE in a multiorbital system without IS are not well understood. In particular, the cooperative roles of the orbital degrees of freedom and lack of IS have not yet been clarified, although their combination will lead to several characteristic properties of the SHE.

To clarify these roles, it is necessary to study the SHE in a multiorbital system without IS by using a model considering correctly both orbital degrees of freedom and IS breaking. It should be noted that since a combination of these leads to completely different results for several electronic properties from the results for a Rashba-type antisymmetric SOI, the correct treatment beyond the Rashba-type antisymmetric SOI is significant for multiorbital systems. Actually, the momen-
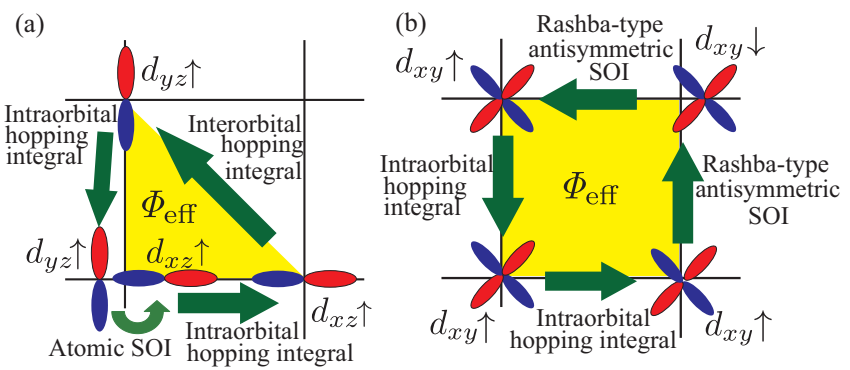

FIG. 1. (Color online) Schematic pictures of some processes generating the effective flux $\Phi_{\text {eff }}$ in (a) the $t_{2 g}$-orbital model with IS 8 and (b) the single-orbital Rashba model without IS 5 . 


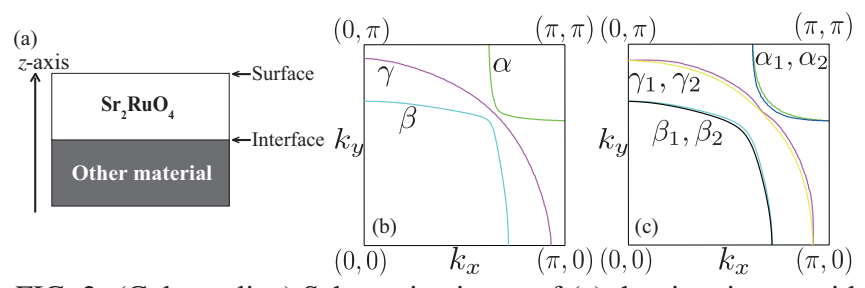

FIG. 2. (Color online) Schematic picture of (a) the situation considered, and Fermi surfaces at (b) $t_{\mathrm{ISB}}=0$ and (c) $t_{\mathrm{ISB}}=0.09 \mathrm{eV}$.

tum dependence of the $\boldsymbol{d}$ vector of a Cooper pair completely differs from that for a Rashba-type antisymmetric SOI due to the difference in the momentum dependence of the antisymmetric SOI; in the correct treatment, the antisymmetric SOI arises from the atomic SOI and the interorbital hopping integral due to local parity mixing, induced by IS breaking 26.27 . Since the momentum dependence of the antisymmetric SOI is important even in discussing the SHE, a study about the SHE using the correct treatment is highly desirable.

In this Rapid Communication, we study the SHE in a $t_{2 g}$ orbital system without IS by using the correct treatment about the orbital degrees of freedom and a lack of IS beyond the Rashba-type antisymmetric SOI, and reveal their cooperative roles in the SHE. In particular, we find that the band anticrossing due to the cooperative roles plays an important role in controlling the magnitude and sign of the spin Hall conductivity (SHC) of a multiorbital system without IS in the presence of dilute nonmagnetic impurities. After discussing the applicability of a similar mechanism, we propose a ubiquitous method to control the magnitude and sign of the Hall effects by using orbital degrees of freedom, IS breaking, and nonmagnetic impurity scattering.

To discuss the SHE in a multiorbital system without IS, we consider a $t_{2 g}$-orbital tight-binding model of the [001] surface or interface of $\mathrm{Sr}_{2} \mathrm{RuO}_{4}$ [Fig. 2]a)] in the presence of dilute nonmagnetic impurities: The Hamiltonian is $H=H_{0}+H_{\mathrm{LS}}+$ $H_{\mathrm{ISB}}+H_{\mathrm{imp}}$, where $H_{0}=\sum_{k} \sum_{a, b} \sum_{\sigma} \varepsilon_{a b}(\boldsymbol{k}) c_{\boldsymbol{k} a \sigma}^{\dagger} c_{\boldsymbol{k} b \sigma}$, $H_{\mathrm{LS}}=\sum_{k} \sum_{a, b} \sum_{\sigma, \sigma^{\prime}} \lambda[\boldsymbol{\ell} \cdot s]_{a \sigma b \sigma^{\prime}} c_{\boldsymbol{k} a \sigma}^{\dagger} c_{\boldsymbol{k} b \sigma^{\prime}}, \quad H_{\mathrm{ISB}}=$ $\sum_{\boldsymbol{k}} \sum_{\sigma}\left[V_{x}(\boldsymbol{k}) c_{\boldsymbol{k} d_{y z} \sigma}^{\dagger} c_{\boldsymbol{k} d_{x y} \sigma}+V_{y}(\boldsymbol{k}) c_{\boldsymbol{k} d_{x z} \sigma}^{\dagger} c_{\boldsymbol{k} d_{x y} \sigma}+\right.$ H.c.], and $H_{\mathrm{imp}}=\sum_{\boldsymbol{k}, \boldsymbol{k}^{\prime}} \sum_{a} \sum_{\sigma} v_{\mathrm{imp}} \sum_{\boldsymbol{R}_{j}} e^{-\mathrm{i}\left(\boldsymbol{k}-\boldsymbol{k}^{\prime}\right) \cdot \boldsymbol{R}_{j}} c_{\boldsymbol{k} a \sigma}^{\dagger} c_{\boldsymbol{k}^{\prime} a \sigma}$, with $a, b=d_{y z}, d_{x z}, d_{x y}$ and $\sigma, \sigma^{\prime}=\uparrow, \downarrow$. Hereafter, we set $c=\hbar=1$ and choose the lattice constant as unity.

$H_{0}$ and $H_{\mathrm{LS}}$ represent the kinetic energy and atomic SOI of $\mathrm{Sr}_{2} \mathrm{RuO}_{4}$, respectively; $\varepsilon_{a b}(\boldsymbol{k})$ are $\varepsilon_{d_{y z} d_{y z}}(\boldsymbol{k})=-2 t_{3} \cos k_{x}-$ $2 t_{2} \cos k_{y}-\mu, \quad \varepsilon_{d_{x z}} d_{x z}(\boldsymbol{k})=-2 t_{2} \cos k_{x}-2 t_{3} \cos k_{y}-\mu$, $\varepsilon_{d_{x y} d_{x y}}(\boldsymbol{k})=-2 t_{1}\left(\cos k_{x}+\cos k_{y}\right)-4 t_{4} \cos k_{x} \cos k_{y}-\mu$, and $\varepsilon_{d_{x z} d_{y z}}(\boldsymbol{k})=\varepsilon_{d_{y z} d_{x z}}(\boldsymbol{k})=4 t_{5} \sin k_{x} \sin k_{y} ; \quad[\boldsymbol{\ell} \cdot \boldsymbol{s}]_{a \sigma b \sigma^{\prime}}$ is $[\ell \cdot s]_{a \sigma b \sigma^{\prime}}=-\frac{1}{2}\left(\delta_{a, d_{y z}} \delta_{b, d_{x y}}-\delta_{b, d_{x y}} \delta_{a, d_{y z}}\right) \delta_{\sigma,-\sigma^{\prime}} \operatorname{sgn}(\sigma)+$ $\frac{i}{2}\left(\delta_{a, d_{x z}} \delta_{b, d_{x y}}-\delta_{b, d_{x y}} \delta_{a, d_{x z}}\right) \delta_{\sigma,-\sigma^{\prime}} \operatorname{sgn}(\sigma)+\frac{i}{2}\left(\delta_{a, d_{y z}} \delta_{b, d_{x z}}-\right.$ $\left.\delta_{b, d_{x z}} \delta_{a, d_{y z}}\right) \delta_{\sigma, \sigma^{\prime}} \operatorname{sgn}(\sigma)$, with $\operatorname{sgn}(\sigma)=+1(-1)$ for $\sigma=\uparrow$ $(\downarrow)$. We choose these parameters so as to reproduce the experimentally observed Fermi surface of $\mathrm{Sr}_{2} \mathrm{RuO}_{4}{ }^{28}$ : $\left(t_{1}, t_{2}, t_{3}, t_{4}, t_{5}, \lambda\right) \quad$ is $\quad(0.45,0.675,0.09,0.18,0.03,0.045)$ $(\mathrm{eV})^{29}$ and the chemical potential $\mu$ is determined so that the number of electrons per site is four.

$H_{\text {ISB }}$ represents the interorbital hopping integral between the $d_{y z / x z}$ and $d_{x y}$ orbitals due to the local parity mixing which is induced by IS breaking near the [001] surface or interface ${ }^{26,27} ; V_{x / y}(\boldsymbol{k})$ is $V_{x / y}(\boldsymbol{k})=2 \mathrm{i}_{\mathrm{ISB}} \sin k_{x / y}$. (For details of the derivation, see the Supplemental Material27.) Note that the second-order perturbation of $H_{\mathrm{LS}}$ and $H_{\mathrm{ISB}}$ gives a Rashbatype antisymmetric SOI when the orbital degeneracy is lifted by the large crystal-electric-field energy 26 (see the Supplemental Material ${ }^{27}$ ). In a case without IS, we set $t_{\mathrm{ISB}}=0.09$ $\mathrm{eV}$ to make $t_{\text {ISB }}$ larger than $\lambda$ and $t_{5}$; as we will show, this condition is essential to obtain the magnitude and sign changes of the SHC. For comparison, we also consider the case at $t_{\mathrm{ISB}}=0$ $\mathrm{eV}$. As shown in the Supplemental Material ${ }^{27}$, to obtain finite intrinsic SHC, only IS breaking is insufficient and finite atomic SOI is essential4.8.

Diagonalizing $H_{\text {band }}=\sum_{k} H_{\text {band }}(\boldsymbol{k})=H_{0}+H_{\mathrm{LS}}+H_{\mathrm{ISB}}$, we obtain the band dispersions $E_{n}(\boldsymbol{k})$ and the unitary matrix $[U(\boldsymbol{k})]_{n \eta}$, where $\eta$ is $\eta \equiv(a, \sigma)$. Comparing the Fermi surfaces at $t_{\mathrm{ISB}}=0$, and $0.09 \mathrm{eV}$ shown in Figs. 2(b) and 2(c), respectively, we see IS breaking causes the spin splitting of the $\alpha, \beta$, and $\gamma$ sheets. (At $t_{\mathrm{ISB}}=0 \mathrm{eV}$, the $\alpha$ and $\beta$ sheets are formed mainly by the $d_{x z}$ and $d_{y z}$ orbitals, and the $\gamma$ sheet is formed mainly by the $d_{x y}$ orbital ${ }^{30}$.) In particular, the most drastic effect of IS breaking is the change of the curvature of the $\gamma$ sheet around $\boldsymbol{k} \sim\left(\frac{2}{3} \pi, \frac{2}{3} \pi\right)$ due to the band anticrossing between the $d_{x y}$ and $d_{x z / y z}$ orbitals. In other words, this band anticrossing causes a change of the main orbital forming the $\gamma$ sheet around $\boldsymbol{k} \sim\left(\frac{2}{3} \pi, \frac{2}{3} \pi\right)$ from the $d_{x y}$ orbital to the $d_{x z / y z}$ orbital. The necessary conditions for this band anticrossing are both that $t_{\text {ISB }}$ is larger than $\lambda$ and that several Fermi surface sheets whose main orbitals are connected by the transverse component of the atomic SOI are close to each other in a certain area of the Brillouin zone.

$H_{\text {imp }}$ represents the local scattering potential due to dilute nonmagnetic impurities. Assuming that the scattering is weak, we can treat its effects by the Born approximation; thus, $H_{\mathrm{imp}}$ affects the SHC through a self-energy correction and a current vertex correction due to the four-point vertex function ${ }^{8.15,25}$. For simplicity, we assume that the main effects of $H_{\text {imp }}$ arise from the band-independent quasiparticle damping $\gamma_{\text {imp }}$ due to a self-energy correction, which is proportional to the nonmagnetic impurity concentration, $n_{\text {imp }}$. We have checked the neglected terms do not qualitatively change the results shown below 31 .

Then, we derive the SHC by the linear-response theory 14,15 , $\sigma_{x y}^{\mathrm{S}}=\sigma_{x y}^{\mathrm{S}(\mathrm{I})}+\sigma_{x y}^{\mathrm{S}(\mathrm{II})}$, where $\sigma_{x y}^{\mathrm{S}(\mathrm{I})}$ is the Fermi surface term,

$$
\begin{aligned}
\sigma_{x y}^{\mathrm{S}(\mathrm{I})}= & \frac{1}{N} \sum_{\boldsymbol{k}} \sum_{\left\{\eta_{1}\right\}} \int_{-\infty}^{\infty} \frac{\mathrm{d} \omega}{2 \pi}\left(-\frac{\partial f(\omega)}{\partial \omega}\right)\left[j_{x}^{\mathrm{S}}(\boldsymbol{k})\right]_{\eta_{1} \eta_{2}} \\
& \times\left[j_{y}^{\mathrm{C}}(\boldsymbol{k})\right]_{\eta_{3} \eta_{4}}\left[G^{\mathrm{R}}(\boldsymbol{k}, \omega)\right]_{\eta_{2} \eta_{3}}\left[G^{\mathrm{A}}(\boldsymbol{k}, \omega)\right]_{\eta_{4} \eta_{1}},
\end{aligned}
$$

and $\sigma_{x y}^{\mathrm{S}(\mathrm{II})}$ is the Fermi sea term,

$$
\begin{aligned}
\sigma_{x y}^{\mathrm{S}(\mathrm{II})}= & \frac{1}{N} \sum_{\boldsymbol{k}} \sum_{\left\{\eta_{1}\right\}} \int_{-\infty}^{\infty} \frac{\mathrm{d} \omega}{2 \pi} f(\omega) \operatorname{Re}\left\{\left[j_{x}^{\mathrm{S}}(\boldsymbol{k})\right]_{\eta_{1} \eta_{2}}\left[j_{y}^{\mathrm{C}}(\boldsymbol{k})\right]_{\eta_{3} \eta_{4}}\right. \\
& \left.\times\left(\left[G^{\mathrm{R}}(\boldsymbol{k}, \omega)\right]_{\eta_{2}} \eta_{3} \frac{\overleftrightarrow{\partial}}{\partial \omega}\left[G^{\mathrm{R}}(\boldsymbol{k}, \omega)\right]_{\eta_{4} \eta_{1}}\right)\right\}
\end{aligned}
$$



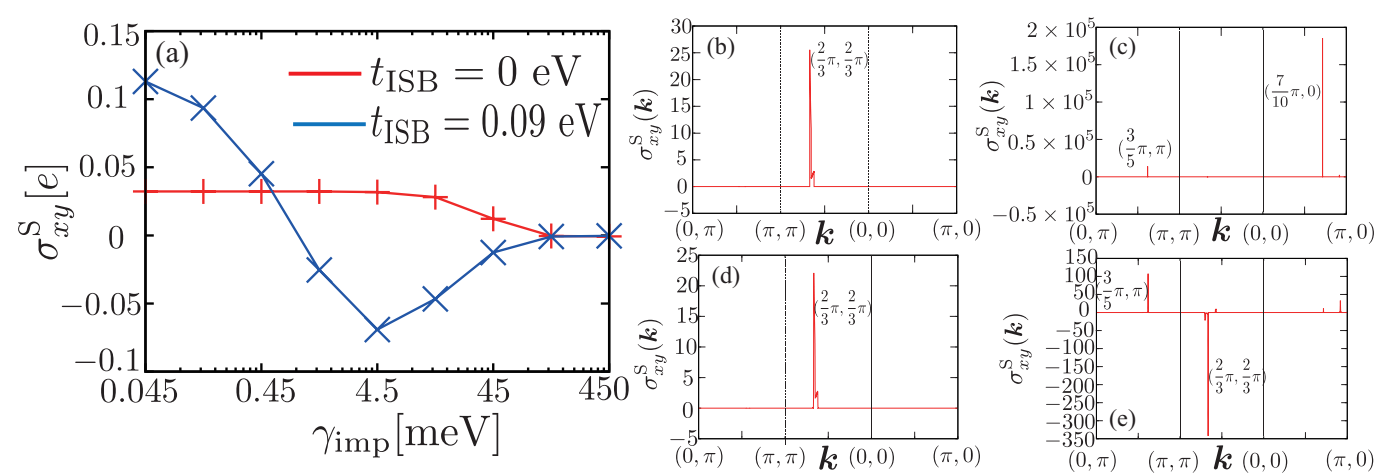

FIG. 3. (Color online) (a) $\gamma_{\mathrm{imp}}$ dependence of $\sigma_{x y}^{\mathrm{S}}$ at $t_{\mathrm{ISB}}=0$ and $0.09 \mathrm{eV}$ and $\boldsymbol{k}$ dependence of $\sigma_{x y}^{\mathrm{S}}(\boldsymbol{k})$ at $(\mathrm{b})\left(\gamma_{\mathrm{imp}}, t_{\mathrm{ISB}}\right)=(0.045 \mathrm{meV}, 0 \mathrm{eV})$, (c) $(0.045 \mathrm{meV}, 0.09 \mathrm{eV}),(\mathrm{d})(4.5 \mathrm{meV}, 0 \mathrm{eV})$, and (e) $(4.5 \mathrm{meV}, 0.09 \mathrm{eV})$.

Here $\sum_{\left\{\eta_{1}\right\}}$ is $\sum_{\left\{\eta_{1}\right\}} \equiv \sum_{\eta_{1}, \eta_{2}, \eta_{3}, \eta_{4}}, f(\omega)$ is the Fermi distribution function, $f(\omega)=\frac{1}{e^{\beta \omega}+1}, G^{\mathrm{R}(\mathrm{A})}(\boldsymbol{k}, \omega)$ is the retarded (advanced) Green's function, $\left[G^{\mathrm{R}(\mathrm{A})}(\boldsymbol{k}, \omega)\right]_{\eta_{1} \eta_{2}}=$ $\sum_{n}\left[U^{\dagger}(\boldsymbol{k})\right]_{\eta_{1} n}\left(\frac{1}{\omega-E_{n}(\boldsymbol{k})+(-) \mathrm{i} \gamma_{\mathrm{imp}}}\right)[U(\boldsymbol{k})]_{n \eta_{2}}, \quad j_{y}^{\mathrm{C}}(\boldsymbol{k}) \quad$ is $\quad$ the charge current operator, $j_{y}^{\mathrm{C}}(\boldsymbol{k}) \equiv-e \frac{\partial H_{\mathrm{band}}(\boldsymbol{k})}{\partial k_{y}}, j_{x}^{\mathrm{S}}(\boldsymbol{k})$ is the spin current operator, $j_{x}^{\mathrm{S}}(\boldsymbol{k}) \equiv \frac{1}{2}\left[s_{z} \frac{\partial H_{\mathrm{band}}(\boldsymbol{k})}{\partial k_{x}}+\frac{\partial H_{\mathrm{band}}(\boldsymbol{k})}{\partial k_{x}} s_{z}\right]$, and $\left(g \frac{\overleftrightarrow{\partial}}{\partial \omega} h\right)$ is $\left(g \frac{\overleftrightarrow{\partial}}{\partial \omega} h\right) \equiv g \frac{\partial h}{\partial \omega}-\frac{\partial g}{\partial \omega} h$. It should be noted, first, that in the clean limit, where $\gamma_{\text {imp }}$ satisfies $\gamma_{\text {imp }} \ll \Delta E(\boldsymbol{k})$ with $\Delta E(\boldsymbol{k})$ being the band splitting giving the dominant contribution to $\sigma_{x y}^{\mathrm{S}}, \sigma_{x y}^{\mathrm{S}}$ is independent of $\gamma_{\mathrm{imp}}$, and is given mainly by the Berry curvature term, part of $\sigma_{x y}^{\mathrm{S}(\mathrm{II})} \underline{15,16}$; second, that in the dirty limit, where $\gamma_{\text {imp }}$ satisfies $\gamma_{\text {imp }} \gg \Delta E(\boldsymbol{k}), \sigma_{x y}^{\mathrm{S}}$ is proportional to $\gamma_{\text {imp }}^{-3}$ and is given mainly by $\sigma_{x y}^{\mathrm{S}(\mathrm{I})} 15,16$.

We turn to results, where we use $20000 \times 20000$ meshes of the first Brillouin zone because this size is necessary to suppress the finite-size effect in a clean region. We first compare the $\gamma_{\text {imp }}$ dependence of $\sigma_{x y}^{\mathrm{S}}$ at $t_{\mathrm{ISB}}=0$ and $0.09 \mathrm{eV}$ in Fig. 3.a). Comparing the results at $t_{\mathrm{ISB}}=0 \mathrm{eV}$ and $0.09 \mathrm{eV}$, we see three changes due to IS breaking: (i) an increase in a clean region $\left(\gamma_{\mathrm{mp}}<0.45 \mathrm{meV}\right)$, (ii) a sign change in a slightly dirty region $\left(0.45 \mathrm{meV} \leq \gamma_{\mathrm{imp}} \leq 45 \mathrm{meV}\right)$, and (iii) the appearance of a minimum at $\gamma_{\text {imp }}=4.5 \mathrm{meV}$. These results suggest that the magnitude and sign of $\sigma_{x y}^{\mathrm{S}}$ can be controlled by using orbital degrees of freedom, IS breaking, and nonmagnetic impurity scattering. As shown in the Supplemental Material27, the above three changes hold in the different values of $t_{\mathrm{ISB}}$ if $t_{\mathrm{ISB}}$ is larger than $\lambda$ and $t_{5}$. Note, first, that in the range of $\gamma_{\text {imp }}$ shown in Fig. $3(\mathrm{a}), \sigma_{x y}^{\mathrm{S}(\mathrm{I})}$ gives the main contribution to $\sigma_{x y}^{\text {S }}$ (see the Supplemental Material ${ }^{27}$ ); and, second, that the residual resistivity estimated by the Drude formula is $0.1 \mu \Omega \mathrm{cm}$ in the clean region and $0.1-10 \mu \Omega \mathrm{cm}$ in the slightly dirty region. To clarify the origins of the above three changes, we analyze the $\boldsymbol{k}$ dependence of $\sigma_{x y}^{\mathrm{S}}(\boldsymbol{k})$, defined as $\sigma_{x y}^{\mathrm{S}}=\frac{1}{N} \sum_{k} \sigma_{x y}^{\mathrm{S}}(\boldsymbol{k})$, at $t_{\mathrm{ISB}}=0$ and $0.09 \mathrm{eV}$. From the results at $\gamma_{\text {imp }}=0.045 \mathrm{meV}$ shown in Figs. 3 (b) and 3 (c), we see as $t_{\mathrm{ISB}}$ changes from 0 to $0.09 \mathrm{eV}$, the main contribution to $\sigma_{x y}^{\mathrm{S}}$ in the clean region changes from the region around $k \sim\left(\frac{2}{3} \pi, \frac{2}{3} \pi\right)$ to the region around $k \sim\left(\frac{7}{10} \pi, 0\right)$. Since the latter main contribution is larger than the former one, change (i) arises from the evolution of the larger positive-sign contribution around $k \sim\left(\frac{7}{10} \pi, 0\right)$. Then, from the results at $\gamma_{\text {imp }}=4.5 \mathrm{meV}$ shown in Figs. 3(d) and 3(e), we see that the change of $t_{\mathrm{ISB}}$ from 0 to $0.09 \mathrm{eV}$ in the slightly dirty region leads to the sign change of the main contribution to $\sigma_{x y}^{\mathrm{S}}$ around $k \sim\left(\frac{2}{3} \pi, \frac{2}{3} \pi\right)$ from positive to negative. Thus, this sign change is the origin of change (ii). Moreover, combining the results in the clean and the slightly dirty regions, we find change (iii) arises from the competition between the opposite-sign contributions around $\boldsymbol{k} \sim\left(\frac{7}{10} \pi, 0\right)$ and $\boldsymbol{k} \sim\left(\frac{2}{3} \pi, \frac{2}{3} \pi\right)$.

In addition, to understand how each $t_{2 g}$ orbital contributes to each $\boldsymbol{k}$ component of $\sigma_{x y}^{\mathrm{S}}(\boldsymbol{k})$ at $t_{\mathrm{ISB}}=0$ and $0.09 \mathrm{eV}$, we analyze orbital-decomposed SHCs, obtained by the equations that the summations with respect to orbital and spin indices in Eqs. (1) and (2) are restricted.

Before the results at $t_{\mathrm{ISB}}=0.09 \mathrm{eV}$, we explain the relation between the main $\boldsymbol{k}$ component of $\sigma_{x y}^{\mathrm{S}}(\boldsymbol{k})$ and each $t_{2 g}$ orbital at $t_{\mathrm{ISB}}=0 \mathrm{eV}$. Considering all the possible orbitaldecomposed SHCs which give the finite contribution to $\sigma_{x y}^{\mathrm{S}}$ and calculating these values, we find that in the clean and the slightly dirty regions, the main contribution to $\sigma_{x y}^{\mathrm{S}}$ arises from the term containing $\left[j_{x}^{\mathrm{S}}(\boldsymbol{k})\right]_{d_{x z} \sigma d_{x z} \sigma}\left[j_{y}^{\mathrm{C}}(\boldsymbol{k})\right]_{d_{y z} \sigma d_{y z} \sigma}$ around $\boldsymbol{k} \sim$ $\left(\frac{2}{3} \pi, \frac{2}{3} \pi\right)$ near the Fermi level; that main contribution arises from the process shown in Fig. 4 a). Although the process shown in Fig. 1 a) contributes to that term, this contribution is smaller than the above contribution [i.e., Fig. [4(a)] in the clean and the slightly dirty regions since the ratio of the magnitude of the former to the latter is roughly proportional to $\gamma_{\text {imp }} / \lambda$ [see Eqs. (6) and (7) in Ref. 8]. Thus, all the $t_{2 g}$ orbitals around $\boldsymbol{k} \sim\left(\frac{2}{3} \pi, \frac{2}{3} \pi\right)$ near the Fermi level play an important role in the SHE at $t_{\mathrm{ISB}}=0 \mathrm{eV}$.

We go on to explain the relation at $t_{\mathrm{ISB}}=0.09$ $\mathrm{eV}$. By using a similar analysis, we find that in the clean region, the main contribution to $\sigma_{x y}^{\mathrm{S}}$ arises from the term containing $\left[j_{x}^{\mathrm{S}}(\boldsymbol{k})\right]_{d_{x z} \sigma d_{x z} \sigma}\left[j_{y}^{\mathrm{C}}(\boldsymbol{k})\right]_{d_{x z} \sigma^{\prime} d_{x y} \sigma^{\prime}}$ or $\left[j_{x}^{\mathrm{S}}(\boldsymbol{k})\right]_{d_{x z} \sigma d_{x z} \sigma}\left[j_{y}^{\mathrm{C}}(\boldsymbol{k})\right]_{d_{x y} \sigma^{\prime} d_{x z} \sigma^{\prime}}$ around $\boldsymbol{k} \sim\left(\frac{7}{10} \pi, 0\right)$ near the Fermi level, whose main contribution arises from the process shown in Fig. 4(b). We also find that in the slightly dirty 

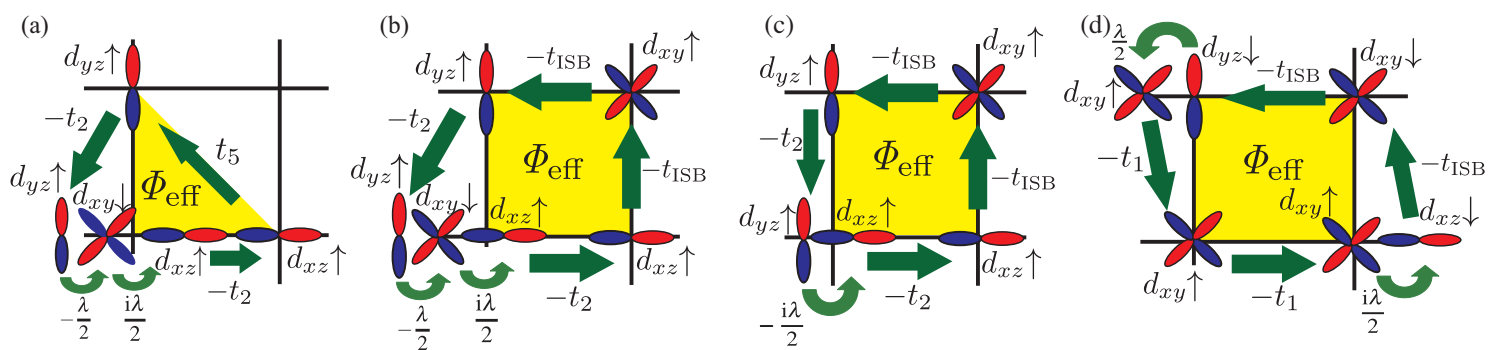

FIG. 4. (Color online) Schematic pictures of the main processes of a spin-up electron (a) at $t_{\mathrm{ISB}}=0 \mathrm{eV}$ in the clean and the slightly dirty region, and at $t_{\mathrm{ISB}}=0.09 \mathrm{eV}$ in (b) the clean and (c) the slightly dirty region, and of (d) an extended Rashba-type process.

region, the main contribution to $\sigma_{x y}^{\mathrm{S}}$ arises from the term containing $\left[j_{x}^{\mathrm{S}}(\boldsymbol{k})\right]_{d_{x z} \sigma d_{x z} \sigma}\left[j_{y}^{\mathrm{C}}(\boldsymbol{k})\right]_{d_{y z} \sigma d_{y z} \sigma}$ around $\boldsymbol{k} \sim\left(\frac{2}{3} \pi, \frac{2}{3} \pi\right)$ near the Fermi level, whose main contribution arises from the process shown in Fig. 4(c). We should note, first, that these processes can be regarded as not the extended Rashba-type ones, but the characteristic ones of a multiorbital system without IS since these completely differ from the extended Rashba-type process such as in Fig. 4(d); and, second, that in the slightly dirty region, although the contribution arising from the process shown in Fig. 4 (a) is of opposite sign to the main contribution [i.e., Fig. 4(c)], the former is smaller due to $t_{\mathrm{ISB}}>t_{5}$ and $t_{\mathrm{ISB}}>\lambda$. Thus, all the $t_{2 g}$ orbitals around $k \sim\left(\frac{7}{10} \pi, 0\right)$ and $\left(\frac{2}{3} \pi, \frac{2}{3} \pi\right)$, which are affected by the spin splitting due to IS breaking, near the Fermi level become important in the SHE at $t_{\mathrm{ISB}}=0.09 \mathrm{eV}$. In particular, the sign change of the contribution around $k \sim\left(\frac{2}{3} \pi, \frac{2}{3} \pi\right)$ due to the band anticrossing and its competition with the opposite-sign contribution around $k \sim\left(\frac{7}{10} \pi, 0\right)$ are vital to obtain the magnitude and sign change of the SHC as a function of $\gamma_{\mathrm{imp}}$.

We emphasize that our mechanism for controlling the magnitude and sign of the SHC is qualitatively different from the mechanism proposed in the single-orbital model $\underline{32}$ with the Rashba- and Dresselhaus-type $\frac{33}{3}$ antisymmetric SOIs. This is because our mechanism does not need a bulk IS breaking which is necessary to obtain a Dresselhaus-type antisymmetric SOI.

We now discuss the applicability of our mechanism for controlling the magnitude and sign of the SHC to other systems. If the following three conditions are satisfied, we can control the magnitude and sign of the SHC in a multiorbital system by introducing IS breaking and tuning $n_{\text {imp }}$. These conditions are, first, that there are several (at least two) same-sign contributions from $\sigma_{x y}^{\mathrm{S}}(\boldsymbol{k})$ at some momenta in a case without IS in the absence of band anticrossing; second, that the band anticrossing occurs at one of these momenta due to a combination of orbital degrees of freedom and IS breaking; and, third, that the value of the band splitting at the momentum where the band anticrossing occurs differs from the values of the band splittings giving the other contributions. If the first and second conditions are satisfied, we have the different-sign components of $\sigma_{x y}^{\mathrm{S}}(\boldsymbol{k})$ at some momenta since the band anticrossing causes the sign change of $\sigma_{x y}^{\mathrm{S}}(\boldsymbol{k})$ at one of these momenta, which is a result of the change of the main orbital of the Fermi surface sheet. In addition, if the third condition is satisfied, we can control the magnitude and sign of $\sigma_{x y}^{\mathrm{S}}(\boldsymbol{k})$ by tuning the value of $\gamma_{\text {imp }}$ through $n_{\text {imp }}$ since an increase of $\gamma_{\text {imp }}$ causes a larger decrease of the contribution of $\sigma_{x y}^{\mathrm{S}}(\boldsymbol{k})$ arising from $\Delta E_{1}(\boldsymbol{k})$ than a decrease of the contribution of $\sigma_{x y}^{\mathrm{S}}(\boldsymbol{k})$ arising from $\Delta E_{2}(\boldsymbol{k})>\Delta E_{1}(\boldsymbol{k}), ; \Delta E_{1}(\boldsymbol{k})\left[\Delta E_{2}(\boldsymbol{k})\right]$ is the band gap at the momentum from which the negative (positive) contribution comes. Since a large value of $t_{\mathrm{ISB}}$ is necessary for the band anticrossing and an increase of the potential of the local parity mixing due to IS breaking enhances $t_{\mathrm{ISB}}$, these three conditions can be realized even in other multiorbital systems by introducing IS breaking and tuning $n_{\text {imp }}$. The surface of $\mathrm{SrTiO}_{3}{ }_{3}^{34.35}$ and the interface between $\mathrm{SrTiO}_{3}$ and $\mathrm{LaAlO}_{3} \underline{36}-\underline{38}$ may be good candidates.

Moreover, a similar mechanism is applicable to other Hall effects such as the anomalous Hall effect ${ }^{16.39}$, since the band anticrossing due to a combination of orbital degrees of freedom and IS breaking drastically affects the main process(es) generating the effective flux.

Thus, our mechanism gives a ubiquitous method to control the magnitude and sign of the response of Hall effects in multiorbital systems without IS.

In summary, we studied the SHE in the $t_{2 g}$-orbital tightbinding model of the [001] surface or interface of $\mathrm{Sr}_{2} \mathrm{RuO}_{4}$ in the presence of dilute nonmagnetic impurities on the basis of the linear-response theory. We found that the SHC shows a magnitude increase and sign change as a function of $\gamma_{\text {imp }}$ when the band anticrossing occurs at $\boldsymbol{k}=\left(\frac{2}{3} \pi, \frac{2}{3} \pi\right)$ due to the cooperative roles of the orbital degrees of freedom and IS breaking, and its contribution to the SHC becomes dominant compared with the contributions from $\sigma_{x y}^{\mathrm{S}}(\boldsymbol{k})$ at the other momenta by increasing $\gamma_{\text {imp }}$. Since a similar situation can be realized in other systems by tuning the potential of the local parity mixing due to IS breaking and $n_{\text {imp }}$, we propose that the magnitude and sign of the response of Hall effects in some multiorbital systems can be controlled by introducing IS breaking and tuning $n_{\text {imp }}$.

\section{ACKNOWLEDGMENTS}

We would like to thank M. Ogata, S. Murakami, and T. Okamoto for fruitful discussions and comments. 
* mizoguchi@hosi.phys.s.u-tokyo.ac.jp

1 M. I. Dyakonov, and V. I. Perel, Sov. Phys. JETP Lett. 13, 467 (1971); M. I. Dyakonov, and V. I. Perel, Phys. Lett. A35, 459 (1971).

2 J. E. Hirsch, Phys. Rev. Lett. 83, 1834 (1999).

3 S. A. Wolf, D. D. Awschalom, R. A. Buhrman, J. M. Daughton, S. von Molnár, M. L. Roukes, A. Y. Chtchelkanova, and D. M. Treger, Science 294, 1488 (2001).

4 S. Murakami, N. Nagaosa, and S. C. Zhang, Science 301, 1348 (2003).

5 J. Sinova, D. Culcer, Q. Niu, N. A. Sinitsyn, T. Jungwirth, and A. H. MacDonald, Phys. Rev. Lett. 92, 126603 (2004).

6 S. Murakami, N. Nagaosa, and S. C. Zhang, Phys. Rev. Lett. 93, 156804 (2004).

7 C. L. Kane, and E. J. Mele, Phys. Rev. Lett. 95, 226801 (2005).

8 H. Kontani, T. Tanaka, D. S. Hirashima, K. Yamada, and J. Inoue, Phys. Rev. Lett. 100, 096601 (2008).

9 J. Smit, Physica 24, 39 (1958).

10 L. Berger, Phys. Rev. B 2, 4559 (1970).

11 A. Crépieux, and P. Bruno, Phys. Rev. B 64, 014416 (2001).

12 S. Pandey, H. Kontani, D. S. Hirashima, R. Arita, and H. Aoki, Phys. Rev. B 86, 060507 (R) (2012).

13 M. Onoda, and N. Nagaosa, J. Phys. Soc. Jpn. 71, 19 (2002).

14 P. Středa, J. Phys. C: Solid State Phys. 15, L717 (1982).

15 T. Tanaka, H. Kontani, M. Naito, T. Naito, D. S. Hirashima, K. Yamada, and J. Inoue, Phys. Rev. B 77, 165117 (2008).

16 H. Kontani, T. Tanaka, and K. Yamada, Phys. Rev. B 75, 184416 (2007).

17 K. Nakamura, R. Arita, and H. Ikeda, Phys. Rev. B 83, 144512 (2011).

18 See, e.g., J. J. Sakurai, Modern Quantum Mechanics (Benjamin/Cummings, Menlo Park, CA, 1985).

19 E. Saitoh, M. Ueda, H. Miyajima, and G. Tatara, Appl. Phys. Lett. 88, 182509 (2006).

20 T. Kimura, Y. Otani, T. Sato, S. Takahashi, and S. Maekawa, Phys. Rev. Lett. 98, 156601 (2007).

21 M. Morota, Y. Niimi, K. Ohnishi, D. H. Wei, T. Tanaka, H. Kontani, T. Kimura, and Y. Otani, Phys. Rev. B 83, 174405 (2011).
22 Y. K. Kato, R. C. Myers, A. C. Gossard, and D. D. Awschalom, Science 306, 1910 (2004).

23 J. Wunderlich, B. Kaestner, J. Sinova, and T. Jungwirth, Phys. Rev. Lett. 94, 047204 (2005).

24 E. I. Rashba, Sov. Phys. Solid State 2, 1109 (1960).

25 J. Inoue, G. E. W. Bauer, and L. W. Molenkamp, Phys. Rev. B 67, 033104 (2003).

26 Y. Yanase, J. Phys. Soc. Jpn. 82, 044711 (2013).

27 See the Supplemental Material for supporting information about a microscopic derivation of $H_{\mathrm{ISB}}$, the correspondence between our model and the single-orbital Rashba model, $\sigma_{x y}^{\mathrm{S}}$ without the atomic SOI with IS breaking, $\gamma_{\mathrm{imp}}$ dependence of $\sigma_{x y}^{\mathrm{S}}$ at other values of $t_{\mathrm{ISB}}$, and the role of the Fermi surface term and Fermi sea term.

28 A. P. Mackenzie, S. R. Julian, A. J. Diver, G. J. McMullan, M. P. Ray, G. G. Lonzarich, Y. Maeno, S. Nishizaki, and T. Fujita, Phys. Rev. Lett. 76, 3786 (1996).

29 N. Arakawa, and M. Ogata, Phys. Rev. B 87, 195110 (2013).

30 I. I. Mazin, and D. J. Singh, Phys. Rev. Lett. 79, 733 (1997).

31 T. Mizoguchi, and N. Arakawa, (unpublished).

32 N. A. Sinitsyn, E. M. Hankiewicz, W. Teizer, and J. Sinova, Phys. Rev. B 70, 081312 (R) (2004).

33 G. Dresselhaus, Phys. Rev. 100, 580 (1955).

34 K. Ueno, S. Nakamura, H. Shimotani, A. Ohtomo, N. Kimura, T. Nojima, H. Aoki, Y. Iwasa, and M. Kawasaki, Nat. Mater. 7, 855 (2008).

35 P. D. C. King, S. McKeown Walker, A. Tamai, A. de la Torre, T. Eknapakul, P. Buaphet, S.-K. Mo, W. Meevasana, M. S. Bahramy, and F. Baumberger, Nat. Commun. 5, 3414 (2014).

36 A. Ohtomo, and H. Y. Hwang, Nature 427, 423 (2004).

37 M. Hirayama, T. Miyake, and M. Imada, J. Phys. Soc. Jpn. 81, 084708 (2012).

38 Y. Nakamura, and Y. Yanase, J. Phys. Soc. Jpn. 82, 083705 (2013).

39 R. Karplus, and J. M. Luttinger, Phys. Rev. 95, 1154 (1954); J. M. Luttinger, Phys. Rev. 112, 739 (1958). 


\title{
Supplemental Material for "Controlling spin Hall effect by using a band anticrossing and nonmagnetic impurity scattering"
}

\author{
T. Mizoguchi* and N. Arakawa \\ Department of Physics, The University of Tokyo, \\ 7-3-1 Hongo, Bunkyo-ku, Tokyo 113-0033, Japan
}

(Dated: March 19, 2021)

\begin{abstract}
In this Supplemental Material, we explain a microscopic derivation of $H_{\mathrm{ISB}}$, derive the Rashba-type antisymmetric spin-orbit interaction from our model in the single-orbital limit, highlight the essential role of the atomic spin-orbit interaction in the intrinsic spin Hall effect, present the $\gamma_{i m p}$ dependence of $\sigma_{x y}^{\mathrm{S}}$ at different values of $t_{\mathrm{ISB}}$, and show the role of the Fermi surface term and Fermi sea term in the range of $\gamma_{\mathrm{imp}}$ considered in the main text.
\end{abstract}

PACS numbers: $72.25 . \mathrm{Ba}, 73.40 .-\mathrm{c}, 74.70 . \mathrm{Pq}$ 


\section{DERIVATION OF $H_{\mathrm{ISB}}$}

In this section, we derive $H_{\text {ISB }}$ microscopically by using the Slater-Koster method ${ }^{1}$ and show that $H_{\mathrm{ISB}}$ originates from the local parity mixings both between the $d_{y z}$ orbital at a $\mathrm{Ru}$ site and the $p_{y}$ orbital at an $\mathrm{O}$ site along $x$ direction and between the $d_{x z}$ orbital at a Ru site and the $p_{x}$ orbital at an $\mathrm{O}$ site along $y$ direction. Since the derivation of the term of $H_{\mathrm{ISB}}$ along $y$ direction is similar way to that along $x$ direction, we explain only the derivation of the term along $x$ direction in the following.

Before deriving the term of $H_{\text {ISB }}$ along $x$ direction, we connect the finite terms of the nearestneighbor (n.n.) hopping integrals of $H_{0}$ along $x$ direction with hopping integrals between the Ru- $t_{2 g}$ orbitals and O-2p orbitals because the understanding of that connection is useful for the derivation of $H_{\text {ISB }}$.

Since $\mathrm{Sr}_{2} \mathrm{RuO}_{4}$ is a quasi-two-dimensional system² ${ }^{2}$, and $\mathrm{Ru}$ sites in an $a b$ plane form a square lattice [see Fig. 1(a)], the main n.n. hopping integrals for the $\mathrm{Ru} t_{2 g}$ orbitals along $x$ direction come from the hopping integrals between the $d_{x z}$ and $p_{z}$ orbitals and between the $d_{x y}$ and $p_{y}$ orbitals: the main hopping integral from $\mathrm{Ru} 1$ to $\mathrm{Ru} 2$ sites becomes

$$
\begin{aligned}
H_{0}^{d_{2} d_{1}} & =\frac{\left(H_{0}^{p d_{2}}\right)^{\dagger} H_{0}^{p d_{1}}}{\Delta_{p d}} \\
& =\hat{d}_{2}^{\dagger}\left(\begin{array}{ccc}
0 & 0 & 0 \\
0 & -\frac{V_{p d \pi}^{2}}{\Delta_{p d}} & 0 \\
0 & 0 & -\frac{V_{p d \pi}^{2}}{\Delta_{p d}}
\end{array}\right) \hat{d}_{1},
\end{aligned}
$$

where $\Delta_{p d}$ is the atomic energy difference between the Ru- $t_{2 g}$ and O- $2 p$ orbitals, and $H_{0}^{p d_{i}}$ repre-

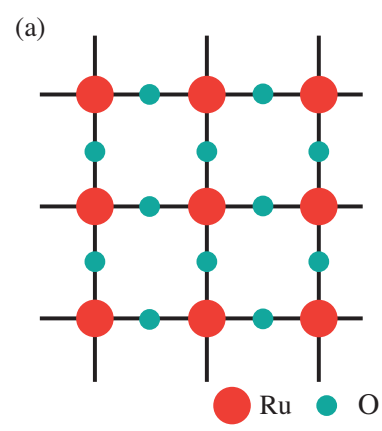

(b) $\mathrm{Ru} 1$

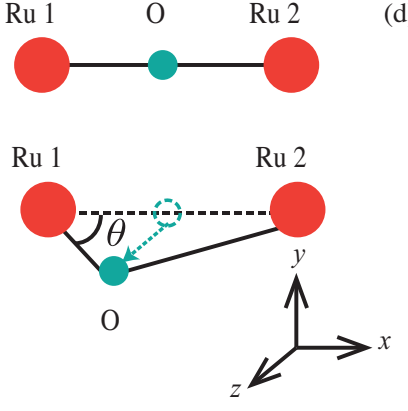

(d) $\mathrm{Ru} 1 \quad \mathrm{O} \quad \mathrm{Ru} 2$

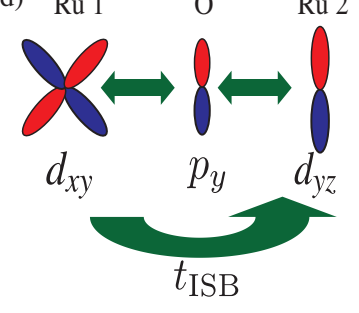

FIG. 1. (Color online) Schematic pictures of (a) an $a b$ plane of $\mathrm{Sr}_{2} \mathrm{RuO}_{4}$, the configuration of a Ru-O-Ru bond (b) with IS and (c) without IS, and (d) an oxygen-mediated process which gives rise to $H_{\text {ISB }}$. 
sents the hopping integrals between the $2 p$ orbitals at an $\mathrm{O}$ site and the $t_{2 g}$ orbitals at a $\mathrm{Ru} i$ sites in the configuration shown in Fig. 1(b), i.e.,

$$
H_{0}^{p d_{1}}=\hat{p}^{\dagger}\left(\begin{array}{ccc}
0 & 0 & 0 \\
0 & 0 & V_{p d \pi} \\
0 & V_{p d \pi} & 0
\end{array}\right) \hat{d}_{1}
$$

and

$$
H_{0}^{p d_{2}}=\hat{p}^{\dagger}\left(\begin{array}{ccc}
0 & 0 & 0 \\
0 & 0 & -V_{p d \pi} \\
0 & -V_{p d \pi} & 0
\end{array}\right) \hat{d}_{2}
$$

with $\hat{p}^{\dagger}=\left(\begin{array}{lll}c_{p_{x}}^{\dagger} & c_{p_{y}}^{\dagger} & c_{p_{z}}^{\dagger}\end{array}\right), \hat{d}_{i}=\left(c_{i, d_{y z}}^{\dagger} c_{i, d_{x z}}^{\dagger} c_{i, d_{x y}}^{\dagger}\right)^{\mathrm{T}}$, and $V_{p d \pi}$, the Slater-Koster parameter ${ }^{1}$ of $\pi$ bonding. Since the matrix elements of $H_{0}^{d_{2} d_{1}}$ and $H_{0}^{d_{1} d_{2}}$ are the same, $H_{0}$ becomes even with respect to momentum [see $\varepsilon_{a b}(\boldsymbol{k})$ of $\hat{H}_{0}$ in the main text]. In Eq. (1), we have neglected the orbital dependence of $\Delta_{p d}$ and $V_{p d \pi}$ for simplicity because the origin and expression of $H_{\text {ISB }}$ are the same except the expression of $t_{\mathrm{ISB}}$ in terms of $\Delta_{p d}$ and $V_{p d \pi}$; if we take account of orbital dependence realized in our model, the expression of $t_{\mathrm{ISB}}$ becomes more complex, although the values of $t_{\mathrm{ISB}}$ along $x$ and $y$ directions remain the same, resulting in the same expression of $H_{\text {ISB }}$. In contrast to the expression of $H_{\mathrm{ISB}}$, we should consider such orbital dependence to obtain the correct expression of $H_{0}$ of our model. Since the aim of this section is microscopic derivation of $H_{\mathrm{ISB}}$, our simplification of $\Delta_{p d}$ and $V_{p d \pi}$ are valid.

However, if inversion symmetry (IS) is broken, the IS breaking may induce several hopping integrals which are prohibited in the presence of IS. In the following, we consider such hopping integrals and show that these lead to $H_{\text {ISB }}$. By creating a [001] surface or an interface of $\mathrm{Sr}_{2} \mathrm{RuO}_{4}$, the IS of $a b$ plane near the surface or the boundary is broken. That IS breaking causes the shifts of $O$ sites along $z$ direction ${ }^{3}$ due to the lack of one of the apical oxygens of a $\mathrm{RuO}_{6}$ octahedral. As a result of those shifts, we obtain the hopping integrals,

$$
\tilde{H}_{p d_{1}}=\hat{p}^{\dagger}\left(\begin{array}{ccc}
0 & \sqrt{3} V_{p d \sigma} \sin \theta \cos ^{2} \theta+V_{p d \pi}\left(1-2 \cos ^{2} \theta\right) \sin \theta & 0 \\
V_{p d \pi} \sin \theta & 0 & V_{p d \pi} \cos \theta \\
0 & \sqrt{3} V_{p d \sigma} \cos \theta \sin ^{2} \theta+V_{p d \pi} \cos \theta\left(1-2 \sin ^{2} \theta\right) & 0
\end{array}\right) \hat{d}_{1},
$$


and

$$
\tilde{H}_{p d_{2}}=\hat{p}^{\dagger}\left(\begin{array}{ccc}
0 & \sqrt{3} V_{p d \sigma} \sin \theta \cos ^{2} \theta+V_{p d \pi}\left(1-2 \cos ^{2} \theta\right) \sin \theta & 0 \\
V_{p d \pi} \sin \theta & 0 & -V_{p d \pi} \cos \theta \\
0 & -\sqrt{3} V_{p d \sigma} \cos \theta \sin ^{2} \theta-V_{p d \pi} \cos \theta\left(1-2 \sin ^{2} \theta\right) & 0
\end{array}\right) \hat{d}_{2}
$$

where $\theta$ is the angle between $x$ axis and the bond between Ru 1 and $\mathrm{O}$ [see Fig. 1(c)], and $V_{p d \sigma}$ is the Slater-Koster parameter of $\sigma$-bonding. Here we neglect the change of $V_{p d \sigma}, V_{p d \pi}$, and $\Delta_{p d}$ due to IS breaking for simplicity; this treatment is sufficient for the purpose of this section because of the similar reason for neglecting the orbital dependence of $\Delta_{p d}$ and $V_{p d \pi}$. Then, to simplify Eqs. (3a) and (3b), we assume that $\theta$ is sufficiently small (i.e., the shift is much smaller than the lattice constant). As a result, we can use the approximation $\cos \theta \sim 1$, and $\sin \theta \sim \theta$, and retain only the lowest order terms with respect to $\theta$. Thus, we have the simple forms of the hopping integrals, $\tilde{H}_{p d_{i}}=H_{0}^{p d_{i}}+H_{p d_{i}}^{\mathrm{ISB}}$, with

$$
H_{p d_{1}}^{\mathrm{ISB}}=\hat{p}^{\dagger}\left(\begin{array}{ccc}
0 & \sqrt{3} V_{p d \sigma} \theta-V_{p d \pi} \theta & 0 \\
V_{p d \pi} \theta & 0 & 0 \\
0 & 0 & 0
\end{array}\right) \hat{d}_{1}
$$

and

$$
H_{p d_{2}}^{\mathrm{ISB}}=\hat{p}^{\dagger}\left(\begin{array}{ccc}
0 & \sqrt{3} V_{p d \sigma} \theta-V_{p d \pi} \theta & 0 \\
V_{p d \pi} \theta & 0 & 0 \\
0 & 0 & 0
\end{array}\right) \hat{d}_{2}
$$

Equations (4) and (5) show that the IS breaking leads to the finite hopping integral which is odd with respect to $z$ finite, although in the presence of the IS, the finite hopping integrals along $x$ direction should be even with respect to $z$. This is the effect of the local parity mixing due to the IS breaking. Thus, the new hopping integrals due to the IS breaking lead to an additional hopping of the $t_{2 g}$ orbitals in combination with the hopping integrals existing in the presence of the IS breaking [see Fig. 1(d)]: that additional hopping integral either from Ru 1 and Ru 2 sites or from 
$\mathrm{Ru} 2$ and $\mathrm{Ru} 1$ sites is, respectively,

$$
\begin{aligned}
H_{\mathrm{ISB}}^{d_{2} d_{1}} & =\frac{\left(H_{0}^{p d_{2}}\right)^{\dagger} H_{\mathrm{ISB}}^{p d_{1}}+\left(H_{\mathrm{ISB}}^{p d_{2}}\right)^{\dagger} H_{0}^{p d_{1}}}{\Delta_{p d}} \\
& =\hat{d}_{2}^{\dagger}\left(\begin{array}{ccc}
0 & 0 & -\frac{2 \theta V_{p d \pi}^{2}}{\Delta_{p d}} \\
0 & 0 & 0 \\
\frac{2 \theta V_{p d \pi}^{2}}{\Delta_{p d}} & 0 & 0
\end{array}\right) \hat{d}_{1} \\
& =\hat{d}_{2}^{\dagger}\left(\begin{array}{ccc}
0 & 0 & -t_{\mathrm{ISB}} \\
0 & 0 & 0 \\
t_{\mathrm{ISB}} & 0 & 0
\end{array}\right) \hat{d}_{1},
\end{aligned}
$$

or

$$
\begin{aligned}
H_{\mathrm{ISB}}^{d_{1} d_{2}} & =\frac{\left(H_{0}^{p d_{1}}\right)^{\dagger} H_{\mathrm{ISB}}^{p d_{2}}+\left(H_{\mathrm{ISB}}^{p d_{1}}\right)^{\dagger} H_{0}^{p d_{2}}}{\Delta_{p d}} \\
& =\hat{d}_{1}^{\dagger}\left(\begin{array}{ccc}
0 & 0 & \frac{2 \theta V_{p d \pi}^{2}}{\Delta_{p d}} \\
0 & 0 & 0 \\
-\frac{2 \theta V_{p d \pi}^{2}}{\Delta_{p d}} & 0 & 0
\end{array}\right) \hat{d}_{2} \\
& =\hat{d}_{1}^{\dagger}\left(\begin{array}{ccc}
0 & 0 & t_{\mathrm{ISB}} \\
0 & 0 & 0 \\
-t_{\mathrm{ISB}} & 0 & 0
\end{array}\right) \hat{d}_{2},
\end{aligned}
$$

where $t_{\mathrm{ISB}}$ is $t_{\mathrm{ISB}}=\frac{2 \theta V_{p d \pi}^{2}}{\Delta_{p d}}$. Since the matrix elements of $H_{\mathrm{ISB}}^{d_{2} d_{1}}$ and $H_{\mathrm{ISB}}^{d_{1} d_{2}}$ are the same except a factor (-1), $H_{\text {ISB }}$ becomes odd with respect to momentum. More precisely, the term of $H_{\text {ISB }}$ along $x$ direction, $H_{\mathrm{ISB} \| x}$, becomes

$$
\begin{aligned}
H_{\mathrm{ISB} \| x} & =t_{\mathrm{ISB}} \sum_{i}\left[\left(c_{\boldsymbol{R}_{i}, y z}^{\dagger} c_{\boldsymbol{R}_{i}+\boldsymbol{e}_{x}, x y}+\left(c_{\boldsymbol{R}_{i}, y z}^{\dagger} c_{\boldsymbol{R}_{i}-\boldsymbol{e}_{x}, x y}\right)+(\text { H.c. })\right]\right. \\
& =2 \mathrm{i} t_{\mathrm{ISB}} \sum_{\boldsymbol{k}} \sin k_{x}\left(c_{\boldsymbol{k} y z}^{\dagger} c_{\boldsymbol{k} x y}-c_{\boldsymbol{k} x y}^{\dagger} c_{\boldsymbol{k} y z}\right),
\end{aligned}
$$

with $e_{x}$ being the unit lattice vector in $x$ direction.

We emphasize that we can straightforwardly extend the above derivation to case of other models. 


\section{DERIVATION OF THE RASHBA-TYPE SPIN-ORBIT INTERACTION}

In this section, we derive the Rashba-type antisymmetric spin-orbit interaction (SOI) ${ }^{4}$ from our model by taking the single-orbital limit and show that the Rashba-type antisymmetric SOI originates from the combination of $H_{\mathrm{ISB}}$, the transverse components of $H_{\mathrm{LS}}$, and the large crystal field splitting.

To derive the Rashba-type antisymmetric SOI, we derive the antisymmetric SOI of our model in the single-orbital limit because the Rashba-type antisymmetric SOI is an effective antisymmetric SOI for a single-orbital system ${ }^{5}$.

To take the single-orbital limit of our model, we add $H_{\mathrm{CF}}$,

$$
H_{\mathrm{CF}}=-\Delta_{\mathrm{CF}} \sum_{i, \sigma} c_{i, d_{x y}, \sigma}^{\dagger} c_{i, d_{x y}, \sigma}
$$

which represents the atomic energy difference between the $d_{x y}$ and $d_{x z / y z}$ orbitals, to the Hamiltonian of our model, and choose $\Delta_{\mathrm{CF}}$ as being much larger than all the hopping integrals and $\lambda$, and put $\mu$ in the range of $-\Delta_{\mathrm{CF}}<\mu<0$. Thus, in this limit, our model reduces to an effective
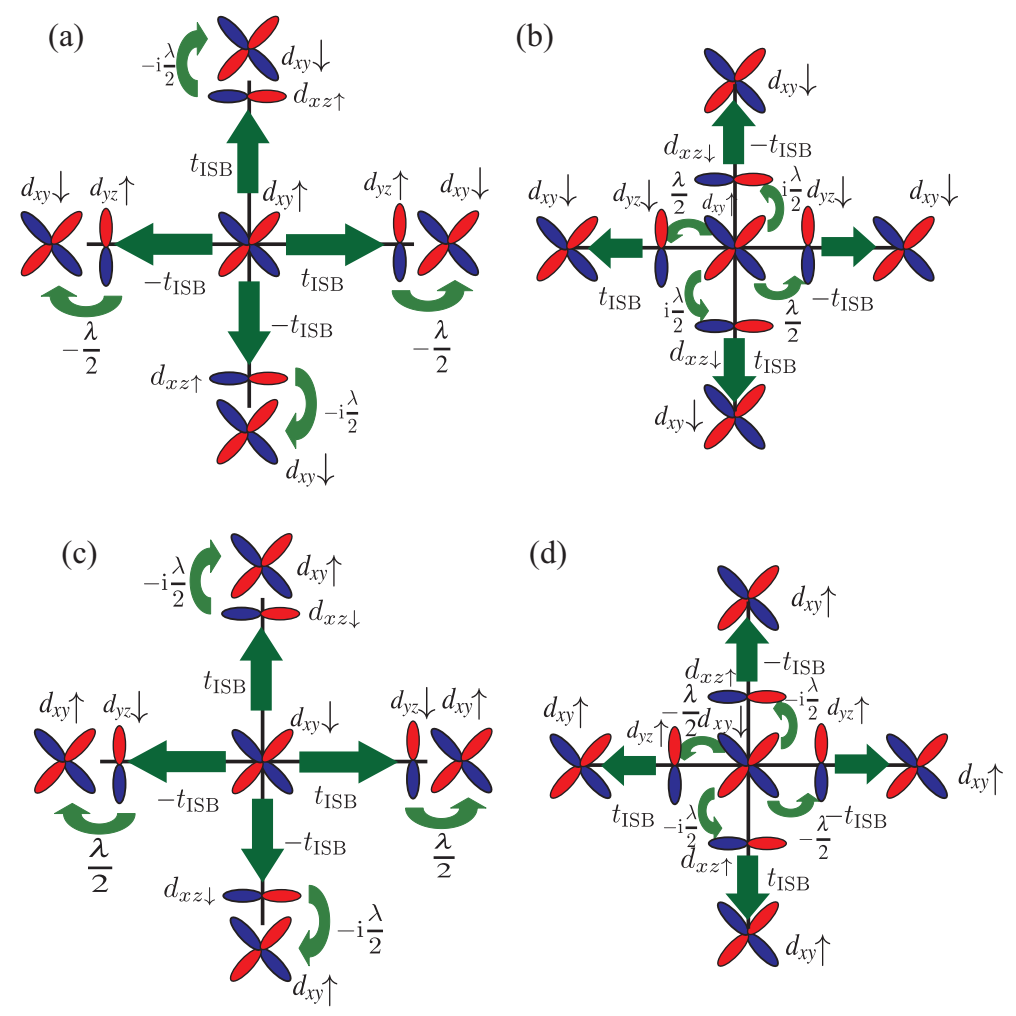

FIG. 2. (Color online) Processes which give rise to the Rashba-type SOI. Here we consider the second-order perturbation using the terms of $H_{\mathrm{ISB}}$ and $H_{\mathrm{LS}}$. 
single-orbital model of the $d_{x y}$ orbital. Because of a much larger value of $\Delta_{\mathrm{CF}}$, we can treat the effects of $H_{\mathrm{ISB}}$ and $H_{\mathrm{LS}}$ as the perturbations against $H_{\mathrm{CF}}$.

Since the lowest-order terms are obtained by using the terms of $H_{\mathrm{ISB}}$ and $H_{\mathrm{LS}}$ each once [for all the possible processes see Figs. 2 (a) $-2(d)]$, we obtain the effective single-orbital antisymmetric SOI in this single-orbital limit:

$$
\begin{aligned}
H_{\mathrm{ASSO}} & =\frac{t_{\mathrm{ISB}} \lambda}{\Delta_{\mathrm{CF}}}\left[\sum_{i}-\left(c_{\boldsymbol{R}_{i}+\boldsymbol{e}_{x}, d_{x y} \downarrow}^{\dagger} c_{\boldsymbol{R}_{i}, d_{x y} \uparrow}-c_{\boldsymbol{R}_{i}-\boldsymbol{e}_{x}, d_{x y} \downarrow}^{\dagger} c_{\boldsymbol{R}_{i}, d_{x y} \uparrow}\right)\right. \\
& -\mathrm{i}\left(c_{\boldsymbol{R}_{i}+\boldsymbol{e}_{y}, d_{x y} \downarrow}^{\dagger} c_{\boldsymbol{R}_{i}, d_{x y} \uparrow}-c_{\boldsymbol{R}_{i}-\boldsymbol{e}_{y}, d_{x y} \downarrow}^{\dagger} c_{\boldsymbol{R}_{i}, d_{x y} \uparrow}\right)+\left(c_{\boldsymbol{R}_{i}+\boldsymbol{e}_{x}, d_{x y} \uparrow}^{\dagger} c_{\boldsymbol{R}_{i}, d_{x y} \downarrow}-c_{\boldsymbol{R}_{i}-\boldsymbol{e}_{x}, d_{x y} \uparrow}^{\dagger} c_{\boldsymbol{R}_{i}, d_{x y} \downarrow}\right) \\
& \left.-\mathrm{i}\left(c_{\boldsymbol{R}_{i}+\boldsymbol{e}_{y}, d_{x y} \uparrow}^{\dagger} c_{\boldsymbol{R}_{i}, d_{x y \downarrow}}-c_{\boldsymbol{R}_{i}-\boldsymbol{e}_{y}, d_{x y} \uparrow}^{\dagger} c_{\boldsymbol{R}_{i}, d_{x y} \downarrow}\right)\right] \\
& =\frac{2 t_{\mathrm{ISB}} \lambda}{\Delta_{\mathrm{CF}}} \sum_{\boldsymbol{k}}\left(c_{\boldsymbol{k} d_{x y} \uparrow}^{\dagger}, c_{\boldsymbol{k} d_{x y} \downarrow}^{\dagger}\right)\left(\begin{array}{cc}
0 & \sin k_{y}+i \sin k_{x} \\
\sin k_{y}-i \sin k_{x} & 0
\end{array}\right)\left(\begin{array}{c}
c_{\boldsymbol{k} d_{x y} \uparrow} \\
c_{\boldsymbol{k} d_{x y} \downarrow}
\end{array}\right)
\end{aligned}
$$

where $e_{y}$ is the unit lattice vector in $y$ direction. This equation shows that $H_{\mathrm{ASSO}}$ corresponds to the Rashba-type antisymmetric SOI with the coupling constant $\alpha_{\text {Rashba }}=\frac{2 t_{\mathrm{ISB}} \lambda}{\Delta_{\mathrm{CF}}}$. Thus, the Rashbatype antisymmetric SOI can be derived from our model in the single-orbital limit by using the parity-mixing hopping and the transverse component of the atomic SOI.

It should be noted that if we do not take the single-orbital limit (i.e., we consider a multiorbital system), we cannot treat the effects of the $H_{\mathrm{ISB}}$ and $H_{\mathrm{LS}}$ as the perturbations, and those effects cannot be described by the simple Rashba-type antisymmetric SOI (even by the sum of the Rashbatype antisymmetric SOI of each orbital). Actually, as we pointed out in the main text, the effects beyond the simple Rashba-type antisymmetric SOI exist in a multiorbital system $\underline{5}$.

Thus, our formalism can describe not only an effective single-orbital system with the Rashbatype antisymmetric SOI but also a multiorbital system with a more complex antisymmetric SOI. In other words, our formalism has a potential for capturing the effects of the combination of orbital degrees of freedom and IS breaking beyond the Rashba-type SOI. Actually, we highlight the importance of such effects in the intrinsic spin Hall effect (SHE) (see the main text).

\section{ESSENTIAL ROLE OF THE ATOMIC SOI IN THE INTRISIC SHE}

In this section, we present the $\gamma_{\mathrm{imp}}$ dependence of $\sigma_{x y}^{\mathrm{S}}$ at $\lambda=0$ and $t_{\mathrm{ISB}}>0$ and highlight the essential role of the atomic SOI in obtaining finite $\sigma_{x y}^{\mathrm{S}}$.

Since the essential role of the atomic SOI in the intrinsic SHE has been confirmed in a multiorbital system with IS ${ }^{6}$, we need to check the role of the atomic SOI in case without IS. 
To check that role, we set $\lambda=0$ with keeping $t_{\mathrm{ISB}}$ finite and calculate $\sigma_{x y}^{\mathrm{S}}$ at several values of $\gamma_{\text {imp }}$ in the same way as the numerical calculation used in the main text. The calculated result presented in Fig. 3 shows that only the IS breaking is insufficient to obtain the finite response of the SHE. [As described in the main text as well as the first section of this Supplemental Material, the effects of the IS breaking are described by the terms of $H_{\text {ISB }}$.] Combining that calculated result with the results presented in Fig. 3 of the main text, we deduce that the atomic SOI is essential to obtain finite response of the intrinsic SHE even in case without IS.

Then, we can provide the brief explanation of the above result of the numerical calculation. If we set $\lambda=0$, the Hamiltonian is decomposed into spin-up and spin-down sectors and their matrix elements are completely the same. In this case, the matrix elements of the spin current for spin-up sector are the same as those for spin-down sector except a factor of $(-1)$, and the matrix elements of the charge current and the Green's function for spin-up sector are completely the same as the corresponding matrix elements for spin-down sector. Thus, the contributions to $\sigma_{x y}^{\mathrm{S}}$ from spin-up and spin-down sectors are exactly cancelled out. Due to this exact cancellation, $\sigma_{x y}^{\mathrm{S}}$ becomes zero for $\lambda=0$ even without the IS.

\section{IV. $\gamma_{\text {imp }}$ DEPENDENCE OF $\sigma_{x y}^{\mathrm{S}}$ AT THE DIFFERENT VALUES OF $t_{\mathrm{ISB}}$}

In this section, we present the results of $\sigma_{x y}^{\mathrm{S}}$ which support our statement about the importance of the band anticrossing in the sign and magnitude change of $\sigma_{x y}^{\mathrm{S}}$.

For a deeper understanding of the role of the band anticrossing due to the IS breaking, we

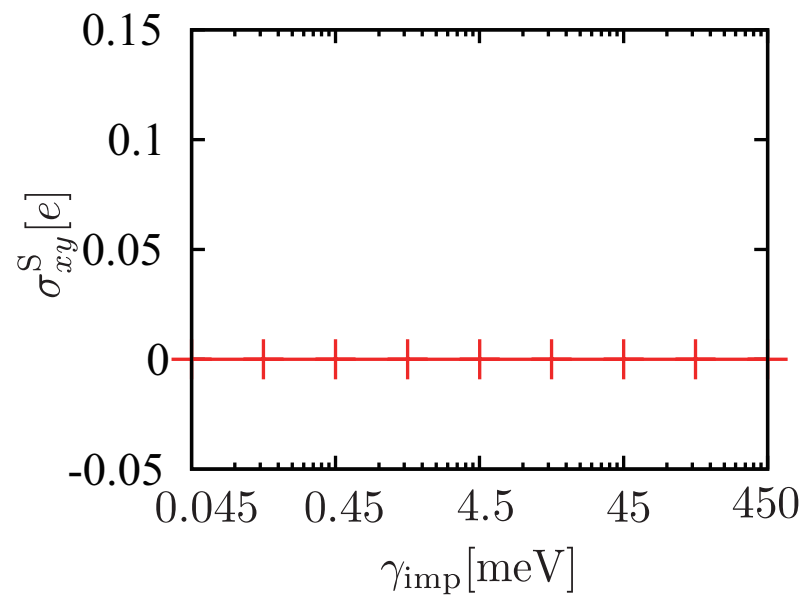

FIG. 3. (Color online) $\gamma_{\mathrm{imp}}$ dependence of $\sigma_{x y}^{\mathrm{S}}$ at $\lambda=0$ and $t_{\mathrm{ISB}}=0.09 \mathrm{eV}$. 

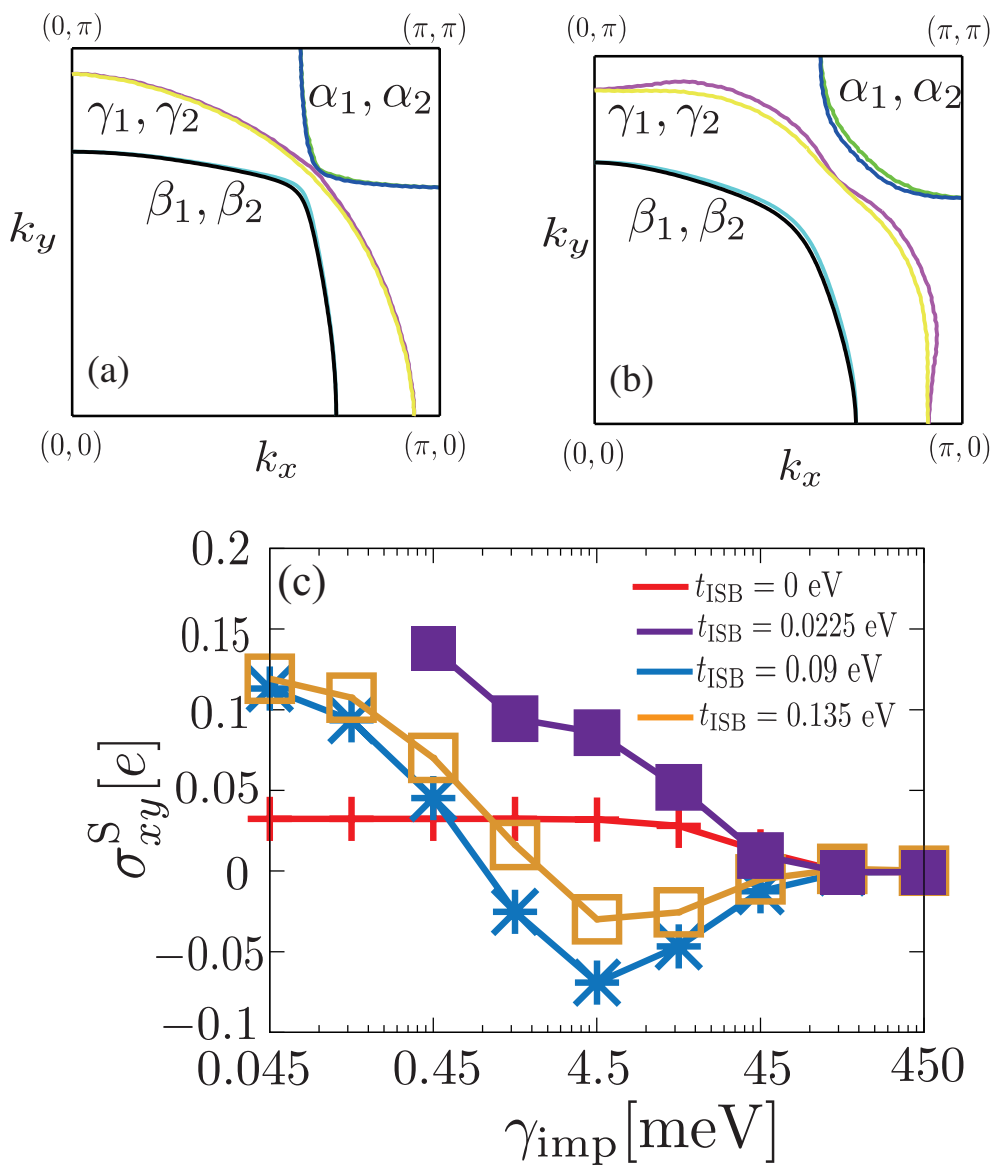

FIG. 4. (Color online) The Fermi surface of (a) $t_{\mathrm{ISB}}=0.0225 \mathrm{eV}$ and (b) $t_{\mathrm{ISB}}=0.135 \mathrm{eV}$, and (c) $\gamma_{\mathrm{imp}}$ dependence of $\sigma_{x y}^{\mathrm{S}}$ at $t_{\mathrm{ISB}}=0,0.0225,0.09$, and $0.135 \mathrm{eV}$.

consider two other cases, case at $t_{\mathrm{ISB}}=0.025 \mathrm{eV}$ and case at $t_{\mathrm{ISB}}=0.135 \mathrm{eV}$, because the band anticrossing occurs only in the latter case due to a large value of $t_{\text {ISB }}$ than the values of $t_{5}$ and $\lambda$. Actually, we see from Figs. 4(a) and 4(b) that the curvature of $\gamma$ sheet around $k=\left(\frac{2 \pi}{3}, \frac{2 \pi}{3}\right)$ does not change at $t_{\mathrm{ISB}}=0.0225 \mathrm{eV}$ and changes at $t_{\mathrm{ISB}}=0.135 \mathrm{eV}$. The change at $t_{\mathrm{ISB}}=0.135 \mathrm{eV}$ is qualitatively the same as that at $t_{\mathrm{ISB}}=0.09 \mathrm{eV}$ [see Fig. 2(c) of the main text].

Then, we calculate the $\gamma_{\mathrm{imp}}$ dependence of $\sigma_{x y}^{\mathrm{S}}$ at $t_{\mathrm{ISB}}=0.0225 \mathrm{eV}$ and $0.135 \mathrm{eV}$ (where the other parameters are the same as case at $t_{\mathrm{ISB}}=0.09 \mathrm{eV}$ ) and compare these results with the results at $t_{\mathrm{ISB}}=0$ and $0.09 \mathrm{eV}$ in Fig. 4(c). Figure 4(c) shows that although the magnitude increases of $\sigma_{x y}^{\mathrm{S}}$ occur at $t_{\mathrm{ISB}}=0.0225 \mathrm{eV}$ and $t_{\mathrm{ISB}}=0.135 \mathrm{eV}$, the sign changes of $\sigma_{x y}^{\mathrm{S}}$ occur only at $t_{\mathrm{ISB}}=0.135$ $\mathrm{eV}$. Those results indicate that we obtain not only the magnitude change of $\sigma_{x y}^{\mathrm{S}}$ but also the sign change of $\sigma_{x y}^{S}$ only in the presence of the band anticrossing due to the IS breaking. Thus, these results support the validity of our mechanism for controlling the magnitude and sign of $\sigma_{x y}^{\mathrm{S}}$ by 
using the band anticrossing and nonmagnetic impurity scattering.

\section{DOMINANCE OF THE FERMI SURFACE TERM OF $\sigma_{x y}^{\mathrm{S}}$}

In this section, we discuss the role of the Fermi surface term and the Fermi sea term in determining $\sigma_{x y}^{\mathrm{S}}$ as a function of $\gamma_{\mathrm{imp}}$ at $t_{\mathrm{ISB}}=0$ and $0.09 \mathrm{eV}$ and show the dominance of the Fermi surface term in the parameter region we consider.

To discuss the role of the Fermi surface term and the Fermi sea term, we compare $\sigma_{x y}^{\mathrm{S}(\mathrm{I})}$ and $\sigma_{x y}^{\mathrm{S}(\mathrm{II})}$ [determined by Eqs. (1) and (2) in the main text, respectively] with $\sigma_{x y}^{\mathrm{S}}$ at several values of $\gamma_{i m p}$ in Figs. 5(a) and 5(b). Those figures show that $\sigma_{x y}^{\mathrm{S}(\mathrm{II})}$ is less dominant and that $\sigma_{x y}^{\mathrm{S}(\mathrm{I})}$ gives the dominant contribution to $\sigma_{x y}^{\mathrm{S}}$.

Thus, the Fermi surface term (which cannot be described by the Berry curvature term, as explained in the main text) is dominant in determining $\sigma_{x y}^{\mathrm{S}}$ in case not only with IS but also without IS in the range of $0.045 \leq \gamma_{\mathrm{imp}} \leq 450(\mathrm{meV})$.

* mizoguchi@ hosi.phys.s.u-tokyo.ac.jp

1 J. C. Slater, and G. F. Koster, Phys. Rev. 94, 1498 (1954).

2 A. P. Mackenzie, S. R. Julian, A. J. Diver, G. J. McMullan, M. P. Ray, G. G. Lonzarich, Y. Maeno, S. Nishizaki, and T. Fujita, Phys. Rev. Lett. 76, 3786 (1996).
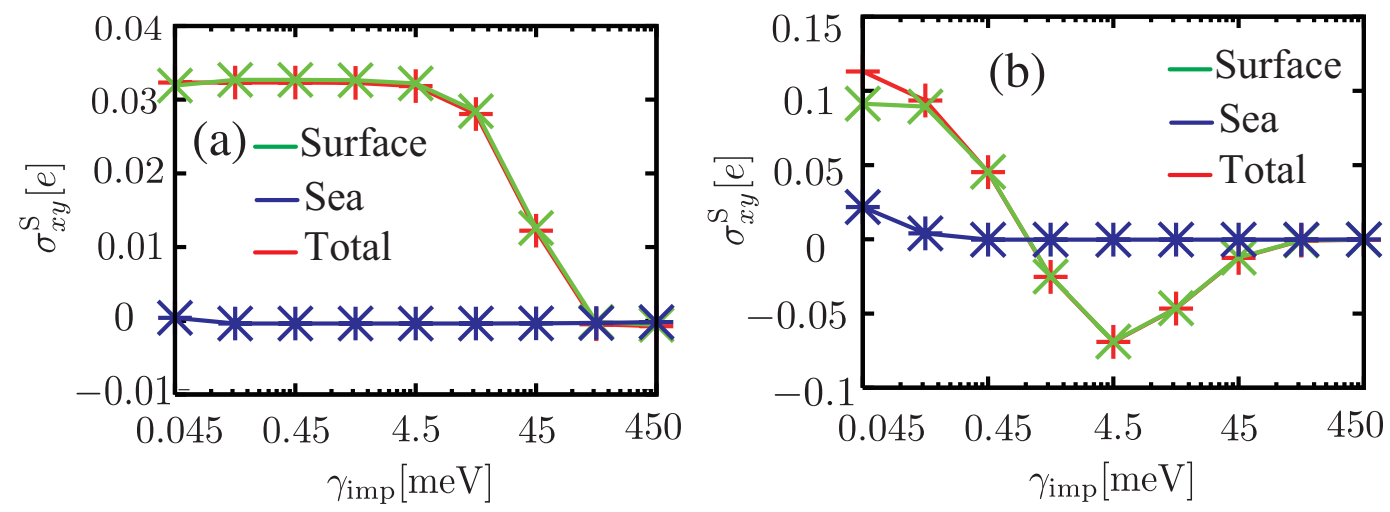

FIG. 5. (Color online) $\gamma_{i m p}$ dependence of the Fermi surface term, $\sigma_{x y}^{\mathrm{S}(\mathrm{I})}$, and the Fermi sea term, $\sigma_{x y}^{\mathrm{S}(\mathrm{II})}$, at (a) $t_{\mathrm{ISB}}=0 \mathrm{eV}$ and (b) $t_{\mathrm{ISB}}=0.09 \mathrm{eV}$. Green lines represent $\sigma_{x y}^{\mathrm{S}(\mathrm{I})}$, blue lines represent $\sigma_{x y}^{\mathrm{S}(\mathrm{II})}$, and red lines represent $\sigma_{x y}^{\mathrm{S}}=\sigma_{x y}^{\mathrm{S}(\mathrm{I})}+\sigma_{x y}^{\mathrm{S}(\mathbb{I I})}$. 
3 M. Onoda, and N. Nagaosa, J. Phys. Soc. Jpn. 71, 19 (2002).

4 E. I. Rashba, Sov. Phys. Solid State 2, 1109 (1960).

5 Y. Yanase, J. Phys. Soc. Jpn. 82, 044711 (2013).

${ }^{6}$ H. Kontani, T. Tanaka, D. S. Hirashima, K. Yamada, and J. Inoue, Phys. Rev. Lett. 100, 096601 (2008). 